\title{
A inserção das atividades do nutricionista no âmbito do PNAE em escolares no município de Picuí-PB
}

The insertion of the activities of the nutritionist within the scope of the PNAE in schoolchildren in

\author{
the municipality of Picuí-PB \\ La inserción de las actividades de la nutricionista en el ámbito del PNAE en escolares del municipio \\ de Picuí-PB
}

Recebido: 07/03/2021 | Revisado: 14/03/2021 | Aceito: 19/03/2021 | Publicado: 26/03/2021

Jair Francisco de Lima Segundo ORCID: https://orcid.org/0000-0002-8683-6065 Universidade Federal de Campina Grande, Brasil E-mail: jairlimasegundo@gmail.com

Maria Luiza Alves Souza

ORCID: https://orcid.org/0000-0003-0157-0585 Universidade Federal de Campina Grande, Brasil

E-mail: marialuiza.mla12@gmail.com

Ana Alice Domingos Pontes

ORCID: https://orcid.org/0000-0002-3781-9074

E-mail: anaalice5430@gmail.com

Universidade Federal de Campina Grande, Brasil

Heloísa Maria Ângelo Jerônimo

ORCID: https://orcid.org/0000-0002-3139-4589 Universidade Federal de Campina Grande, Brasil E-mail: helogero@yahoo.com.b

Jefferson Carneiro de Barros ORCID: https://orcid.org/0000-0002-3558-796X Universidade Federal de Campina Grande, Brasil E-mail: jefferson.carneiro@professor.ufcg.edu.br

Ediane Deijaly dos Santos

ORCID: https://orcid.org/0000-0003-1597-7984 Prefeitura Municipal de Picuí, Paraíba, Brasil

E-mail: Deijaly@hotmail.com

Angélica Ferreira Barros

ORCID: https://orcid.org/0000-0003-1402-5084

Prefeitura Municipal de Picuí, Paraíba, Brasil

E-mail: barros.angelica.nutri@gmail.com

\begin{abstract}
Resumo
Sendo um dos mais antigos programas públicos de complementação alimentar, o Programa Nacional de Alimentação Escolar (PNAE) desfruta de propostas como a suplementação das necessidades diárias dos escolares matriculados, com o objetivo de garantir a Segurança Alimentar e Nutricional (SAN) e colaborar para a formação de bons hábitos alimentares. A obesidade e/ou sobrepeso associam-se com a quantidade e a qualidade da ingestão alimentar de crianças, fase essa onde a mesma não é totalmente independente e não tem autonomia em suas escolhas, incluindo as suas preferências. O estudo tem o objetivo de realizar e analisar antropometria para identificar agravos à saúde relacionados com a alimentação das crianças. A mesma pesquisa também objetivou identificar as porcentagens de aceitação dos cardápios, com o intuito de reconhecer prováveis inadequações sensoriais nas refeições oferecidas. Os resultados obtidos retrataram características da transição nutricional, que é marcada pelo aumento do indicador de sobrepeso/obesidade, tornando-se necessário atentar para o fato de que, mesmo que as porcentagens de eutrofia tenham se sobressaído em comparação as outras classificações, não deve ser descartada a presença de crianças abaixo ou acima do peso e/ou altura recomendados. A avaliação sensorial mostrou que a preparação não foi bem aceita obtendo nível médio de adesão e da mesma forma na creche não constatou-se boa aceitabilidade em função das características bem marcantes do prato servido, sendo uma delas o próprio sabor do ingrediente principal da refeição. Conclui-se então que os escolares necessitam de ações voltadas a educação nutricional e o cardápio servido deve ser repensado.
\end{abstract}

Palavras-chave: Alimentação escolar; Avaliação nutricional; Teste de aceitabilidade. 


\begin{abstract}
Being one of the oldest public food complementation programs, the National School Feeding Program (PNAE) enjoys proposals such as supplementation of the daily needs of enrolled students, with the objective of ensuring Food and Nutrition Security (SAN) and collaborating for the formation of good eating habits. Obesity and/or overweight are associated with the quantity and quality of children's food intake, a phase where it is not totally independent and has no autonomy in its choices, including their preferences. The study aims to perform and analyze anthropometry to identify health problems related to children's diet. The same research also aimed to identify the percentages of acceptance of the menus, in order to recognize probable sensory inadequacies in the meals offered. The results obtained portrayed characteristics of the nutritional transition, which is marked by the increase in the overweight/obesity indicator, making it necessary to pay close to the fact that, even if the percentages of eutrophy have increased in comparison to the other classifications, the presence of children under or above the recommended weight and/or height should not be ruled out. The sensory evaluation showed that the preparation was not well accepted obtaining the average level of adesand similarly in the daycare center, it was not a good acceptability due to the well-marked characteristics of the dish served, one of which is the taste of the main ingredient of the meal. It is concluded that the students need actions aimed at nutritional education and the menu served should be rethought.
\end{abstract}

Keywords: School feeding; Nutritional assessment; Acceptability test.

\title{
Resumen
}

Al ser uno de los programas de complementación de alimentos públicos más antiguos, el Programa Nacional de Alimentación Escolar (PNAE) disfruta de propuestas como la complementación de las necesidades diarias de los estudiantes inscritos, con el objetivo de garantizar la Seguridad Alimentaria y Nutricional (SAN) y colaborar para la formación de buenos hábitos alimenticios. La obesidad y/o el sobrepeso están asociados con la cantidad y calidad de la ingesta de alimentos infantiles, una fase en la que no es totalmente independiente y no tiene autonomía en sus elecciones, incluidas sus preferencias. El estudio tiene como objetivo realizar y analizar la antropometría para identificar problemas de salud relacionados con la dieta de los niños. La misma investigación también tenía como objetivo identificar los porcentajes de aceptación de los menús, con el fin de reconocer probables insuficiencias sensoriales en las comidas ofrecidas. Los resultados obtenidos retrataron las características de la transición nutricional, que está marcada por el aumento del indicador de sobrepeso/obesidad, por lo que es necesario pagar cerca del hecho de que, incluso si los porcentajes de eutrofia han aumentado en comparación con las otras clasificaciones, no debe descartarse la presencia de niños menores o por encima del peso y/o estatura recomendados. La evaluación sensorial mostró que la preparación no fue bien aceptada obteniendo el nivel promedio de adesand de manera similar en la guardería, no fue una buena aceptabilidad debido a las características bien marcadas del plato servido, uno de los cuales es el sabor del ingrediente principal de la comida. Se concluye que los estudiantes necesitan acciones dirigidas a la educación nutricional y el menú servido debe ser repensado.

Palabras clave: Alimentación escolar; Evaluación nutricional; Prueba de aceptabilidad.

\section{Introduçãa}

Sendo um dos mais antigos programas públicos de complementação alimentar, o Programa Nacional de Alimentação Escolar (PNAE) desfruta de propostas como a suplementação das necessidades nutricionais diárias dos escolares matriculados, com o objetivo de garantir a Segurança Alimentar e Nutricional (SAN) e colaborar para a formação de bons hábitos alimentares. Este programa tem como beneficiários alunos de toda a educação básica de escolas públicas, filantrópicas e entidades comunitárias conveniadas com o poder público. O Fundo Nacional de Desenvolvimento da Educação (FNDE) é quem gerencia e transfere, em caráter suplementar, recursos financeiros aos estados, ao Distrito Federal e aos municípios, entretanto, os entes federativos têm de participar com contraparte financeiro, manutenção das infraestruturas das escolas, dos recursos humanos da unidade de alimentação escolar e das ações de educação alimentar e nutricional (EAN) (Rocha et al., 2018).

Ao longo dos anos, o Brasil através do PNAE sempre manteve e um foco em melhorar as refeições das crianças em idade escolar através da obrigatoriedade de que, 70\% dos recursos financeiros oferecidos às entidades executoras, sejam aplicados unicamente em produtos básicos para a produção da merenda. A existência do nutricionista como Responsável Técnico (RT) do programa são exemplos de atuações que refletem fortemente no progresso da qualidade da alimentação oferecida aos escolares que este programa assiste (Facchini \& Campagnolo, 2020).

O estado nutricional de obesidade e/ou sobrepeso associa-se com a quantidade e a qualidade da ingestão alimentar de crianças, fase essa onde a mesma não é totalmente independente e não tem autonomia em suas escolhas, incluindo as preferências 
alimentares. Considerado um período chave, os anos escolares são vistos como o centro das intervenções nutricionais para a prevenção de desarmonias nutricionais e a promoção de hábitos de vida saudáveis. Sendo o local onde os estudantes passam boa parte do tempo, a escola, é um ambiente sublime para a execução de atividades de EAN, com possibilidades além da aprendizagem, mas também da prática e reforço de hábitos de vida e comportamentos saudáveis (Ferreira et al., 2020).

Não só a educação nutricional é importante nesse contexto, a avaliação do estado nutricional de crianças é um potente parâmetro de saúde e qualidade dos alunos, corroborando para identificação do modelo de desenvolvimento dos seus hábitos alimentares. O cenário de amadurecimento da criança é um forte indicativo das prováveis influências que o meio ambiente pode ter no crescimento infantil. Pesquisas revelam que as crianças de classes socioeconômicas distintas, diferenciam-se no tamanho e no progresso de crescimento, em praticamente todas as sociedades (Souza et al., 2020).

Levando em consideração toda a temática abordada percebe-se que é de grande importância a aplicação de intervenções que intensifiquem a vigilância da segurança alimentar e nutricional de alunos, sendo assim, a pesquisa em questão tem o objetivo de avaliar o estado nutricional de escolares com a finalidade de observar possíveis agravos à saúde no que se refere a alimentação e nutrição dos mesmos. O mesmo estudo também objetivou identificar as porcentagens de aceitação das refeições oferecidas em ambientes escolares, a fim de reconhecer prováveis inadequações sensoriais nas refeições oferecidas.

\section{Metodologia}

\subsection{Tipo de estudo}

Do ponto de vista de sua natureza, o artigo refere-se a uma pesquisa aplicada, que tem o intuito de conceber discernimento sobre o estado nutricional e nível de aceitabilidade em refeições de uma parcela de discentes de uma determinada localidade do município em questão, assim sendo, o estudo permite aperfeiçoar conhecimentos sobre essa população podendo intervir com a mais preferível funcionalidade técnica voltada à resolutividade de questões singulares, como desnutrição, obesidade e baixa adesão ao cardápio, abrangendo assim apreciações de relevância local.

No tocante aos seus objetivos, a pesquisa em foco possui cunho descritivo utilizando apenas registro das circunstâncias analisadas sem intervir nelas, isto é, o estudo observou, registrou, analisou e ordenou dados, sem manipulá-los, isto é, sem intercessão dos profissionais envolvidos com a finalidade de encontrar a frequência com que um fato ocorre, sua natureza, suas características, causas, relações com outros fatos. Assim, para coletar tais dados, utilizou-se de técnicas específicas, dentre as quais se destacam a entrevista coletiva com questionário breve e a observação.

A investigação pretendeu detalhar os dados antropométricos e sensoriais dos alunos de ambos turnos e instituições, com a finalidade de estabelecer possíveis relações entre as variantes em razão de algum fato que possa explicar tais achados, seja ele social, genético ou fisiológico. Abrangeu a utilização de técnicas padronizadas de coleta de dados como aferição de peso e altura, além da contabilidade de aceitação das refeições. Admite-se, então como as únicas formas de levantamento de dados (Prodanov \& de Freitas, 2013)

Quando se refere aos procedimentos técnicos utilizados, este estudo teve a finalidade de realizar um levantamento transversal de caráter quantitativo utilizando da investigação prática de características corporais de indivíduos na finalidade de descobrir ou levantar possíveis hipóteses que expliquem tal achado. Em função da análise sensorial, procedemos à solicitação de informações a um grupo significativo de alunos acerca das refeições disponibilizadas na escola (Pereira et al. 2018).

Por fim, do ponto de vista da abordagem do problema a pesquisa teve particularidade quantitativa considerando que todos os dados podem ser quantificáveis, o que significa transpor os números em pareceres e em informações para catalogá-las e examiná-las, requerendo a utilização de artifícios e de métodos estatísticos como porcentagem, média e outros. (Prodanov \& de Freitas, 2013). 


\subsection{Local de execução}

A pesquisa foi realizada na Escola Municipal de Ensino Fundamental Governador Flávio Ribeiro e na Creche Municipal Marivalda Cassimiro dos Santos. As instituições de ensino são localizadas nos bairros Monte Santo e Cenecista, respectivamente, no município de Picuí que é situado na mesorregião da Borborema e na microrregião do Seridó Oriental Paraibano, situado na Região Geográfica Imediata de Cuité-Nova Floresta. De acordo com o censo realizado pelo IBGE (Instituto Brasileiro de Geografia e Estatística) no ano de 2010, sua população era de 18.222 habitantes, enquanto que que sua estimativa de 2019 era de 18.703 habitantes com área territorial de $661 \mathrm{~km}^{2}$ e Índice de Desenvolvimento Humano Municipal de 0,60, sendo considerado médio para os níveis de classificação do Programa das Nações Unidas para o Desenvolvimento (IBGE, 2020).

\subsection{Avaliação antropométrica}

\subsubsection{Ensino fundamental 1}

Os dados antropométricos foram coletados por alunos de graduação em nutrição, da Universidade Federal de Campina Grande (UFCG). Os alunos foram treinados para a padronização das medidas antropométricas pelos professores responsáveis pela disciplina de prática clínica do curso. A estatura dos escolares, foi medida por meio de uma fita métrica que foi anexada, com fita adesiva, na parede sem rodapé, da sala de multimídias pertencente a escola. As medições foram realizadas em triplicatas, e a medida final resultou da medição que se repetiu. As crianças foram pesadas utilizando balança eletrônica de plataforma média da marca Multilaser com capacidade máxima de $180 \mathrm{~kg}$ e sensibilidade de $100 \mathrm{~g}$, a mesma foi colocada em local adequado, seguindo as recomendações técnicas do Ministério da Saúde. A avaliação foi realizada apenas em um dia no mês de setembro, no período da manhã e da tarde.

Utilizaram-se os índices antropométricos Peso/Idade (P/I), Altura/Idade (A/I) e Índice de Massa Corporal/Idade (IMC/I). O estado nutricional das crianças foi expresso em escore-Z de acordo com o padrão de referência do Multicentre Growth Study (Who Reference 5-19 years) atualmente recomendado pela Organização Mundial da Saúde (OMS) (WHO, 2008).

Os cálculos foram realizados com a utilização do programa "WHO Anthro Plus 2009". Para a definição de baixo peso e baixa estatura foi adotado o ponto de corte P/I e E/I < Escore-z -2; para o peso e estatura adequada, o ponto de corte foi $\geq$ Escore-z +2 e $\geq$ Escore-z -2, respectivamente. Já para a definição do diagnóstico nutricional segundo o índice IMC/I, utilizou-se os seguintes pontos de corte: IMC/I < Escore-z -2, para magreza; > Escore-z - 2 e < Escore- $\mathrm{z}+2$, para eutrófico; > Escore- $\mathrm{z}+1 \mathrm{e}$ < Escore-z+2, para sobrepeso; > Escore-z +2 e <Escore-z +3, para obesidade; e > Escore-z +3 para obesidade grave (Brasil, 2011). A digitação dos dados foi realizada imediatamente após a coleta da informação, em planilhas do programa Excel (Microsoft Inc., Estados Unidos, 2007) com opções de formatação e validação para a diminuição de erros.

\subsubsection{Creche}

Foi realizada também, na semana seguinte, avaliação antropométrica das crianças que frequentavam a creche da cidade, envolvendo peso, altura e idade individualizados, a fim de obter seu possível estado nutricional, dispondo da mesma forma os dados nos programas da OMS: "WHO Anthro 2007" e "WHO Anthro Plus 2009", classificando-os assim, em adequado ou inadequado. Os indicadores correspondentes a classificação das crianças foram P/I, E/I e Peso/Estatura (P/E), preconizados como índices antropométricos adotados pela Vigilância Nutricional para a faixa etária em questão (Brasil, 2011).

A creche atende crianças com idade entre 2 e 6 anos, com turmas até o pré-escolar. A medição e pesagem das mesmas foi realizada também em um único dia, nos turnos da manhã e tarde no horário de suas atividades escolares. Materiais como fita métrica inflexível com medida até $150 \mathrm{~cm}$, fixada nas portas de cada sala, que eram rentes ao chão, e balança portátil digital de vidro "Relaxmedic", com capacidade máxima de $180 \mathrm{~kg}$, foram utilizados como base para a obtenção das medidas (peso e altura) 
antropométricas necessárias. Para medição de ambos, foi pedido as crianças que retirassem seus sapatos e meias quando presentes.

Foram disponibilizados ainda os nomes e respectivas datas de nascimento de cada aluno pelas responsáveis do local, com o intuito de mensurar a idade em anos e meses. Para preservar a identidade das crianças, serão dispostos no presente trabalho apenas a média dos resultados obtidos. Souza et al. (2017) e Oliveira et al. (2017) utilizaram-se de metodologias semelhantes para a obtenção de seus resultados, porém fazendo a comparação entre as Curvas de Crescimento da Organização Mundial da Saúde - OMS e os softwares.

\subsection{Teste de aceitabilidade}

\subsubsection{Ensino fundamental 1}

O teste de aceitabilidade é o conjunto de procedimentos metodológicos, reconhecidos cientificamente, com a finalidade de medir o índice de aceitabilidade para alimentos e preparo dos cardápios da alimentação escolar. Esse teste está entre os métodos sensoriais afetivos recomendados para o PNAE, utilizando a escala hedônica que é um instrumento validado, no que se resume a avaliação de um produto - no caso, o cardápio - a partir de uma escala gradativa que representa a aceitabilidade do aluno (UNIFESP, 2010).

Para a aplicabilidade do instrumento, nas séries do $1^{\circ}$ ao $5^{\circ}$ ano, foram confeccionadas placas utilizando a forma facial que se encontra na ficha de escala hedônica facial mista do "Manual para aplicação dos testes de aceitabilidade no Programa Nacional de Alimentação Escolar - PNAE” (2017). Após o término do recreio, os alunos supervisionados pela nutricionista, explicaram para todas as turmas como seria a aplicabilidade do teste e como cada um participaria. A participação dos mesmos se dava pelo levantamento da mão, quando era exposta cada plaquinha e era realizada a contagem de quantos alunos levantavam a mão. A avaliação foi realizada apenas em um dia no mês de outubro, no período da manhã e da tarde, referente a uma única preparação (UNIFESP, 2010).

Após a coleta de dados, foram analisados os índices de adesão e de aceitação. Índice de Adesão - o cálculo de adesão a preparação do dia foi realizado utilizando-se a proporção: número de estudantes que consumiram a refeição multiplicado por 100, dividido pelo número de estudantes presentes na escola. Como ponto de corte do índice de adesão, considerou-se o percentual mínimo de 85\% com o indicativo de uma adesão alta a preparação. Índice de Aceitação - o cálculo de aceitação dos alunos em relação ao cardápio do dia foi realizado a partir da soma dos votos das cartelas "gostei extremamente" e "gostei moderadamente" multiplicada por 100 e dividida pelo número de alunos que realizaram a refeição. Considerou-se o percentual mínimo de 85\% como ponto de corte indicativo de boa aceitação do cardápio do dia oferecido na escola (UNIFESP, 2010).

Os dados coletados foram digitados no Programa Excel (Microsoft Inc., Estados Unidos, 2007). Para análise dos resultados, foram utilizados os dados totais, dos dois turnos.

\subsubsection{Creche}

Foi aplicado o teste de aceitabilidade de uma das refeições presentes no cardápio usual, composta por arroz e proteína texturizada de soja, com adição de um sachê de $1 \mathrm{~g}$ de suplemento sem sabor NutriSUS, colocado diretamente em cada prato após montagem, pelas manipuladoras de alimento. Os sachês são compostos por um total de 15 micronutrientes, dispostos em vitaminas e minerais, como ferro, vitamina A e B12, que são acrescidos a refeição já pronta para a criança, individualmente. As crianças contempladas com essa estratégia, configuram-se nas matriculadas em creches que participam do Programa Saúde na Escola (PSE), com faixa etária até 5 anos de idade, e com a devida autorização dos pais ou responsáveis. As recomendações de administração estão dispostas no "NutriSUS - Estratégia de fortificação da alimentação infantil com micronutrientes (vitaminas e minerais) em pó: manual operacional" do Ministério da Saúde (MS) (Brasil, 2015). 
A princípio, a ideia também foi de realizar a escala-hedônica por meio de placas com a forma facial, porém, ao ser iniciado o teste, foi visto que as crianças não conseguiram responder com o uso das placas que indicam se elas "gostam" ou "não gostam" da refeição servida, representada por uma expressão feliz e triste, respectivamente. Isso ocorreu possivelmente pelo fato de possuírem, em sua grande maioria, faixa etária menor ou igual a 6 anos de idade, dificultando assim, sua compreensão. Nesse caso, foi necessário adaptar o teste, pedindo apenas que levantassem as mãos as crianças que gostaram da refeição, e posteriormente levantassem as mãos as crianças que não gostaram, perguntando ao final os motivos de não se agradarem do prato servido. Levando-se em consideração ainda, que nem todas as crianças consumiram a refeição por opção, e algumas repetiram.

Além disso, de acordo com o "Manual para Aplicação dos Testes de Aceitabilidade no Programa Nacional de Alimentação Escolar - PNAE” (2017), sabe-se que há limitações e até invalidez dos testes de aceitabilidade em creches, pelo fato de as crianças estarem, muitas vezes, em fase de conhecimento dos alimentos e possuírem tendência a rejeitá-los em seus primeiros contatos, sendo necessárias aproximadamente oito a dez exposições ao mesmo alimento, de forma gradual para avaliar sua aceitabilidade. Mesmo com a possibilidade que os testes de aceitabilidade possuem de contemplar crianças pertencentes a pré-escola, correspondendo a algumas turmas da creche em questão, a adaptação do teste foi a opção mais viável.

\subsection{Análise estatística}

Para o teste de aceitabilidade foi descrito o número de respostas para cada expressão facial (carinha) da escala apresentada na ficha, para assim ser calculado a porcentagem de expressão (carinha), como podemos observar na Figura 1.

Figura 1 - Modelo de ficha de escala hedônica facial mista.

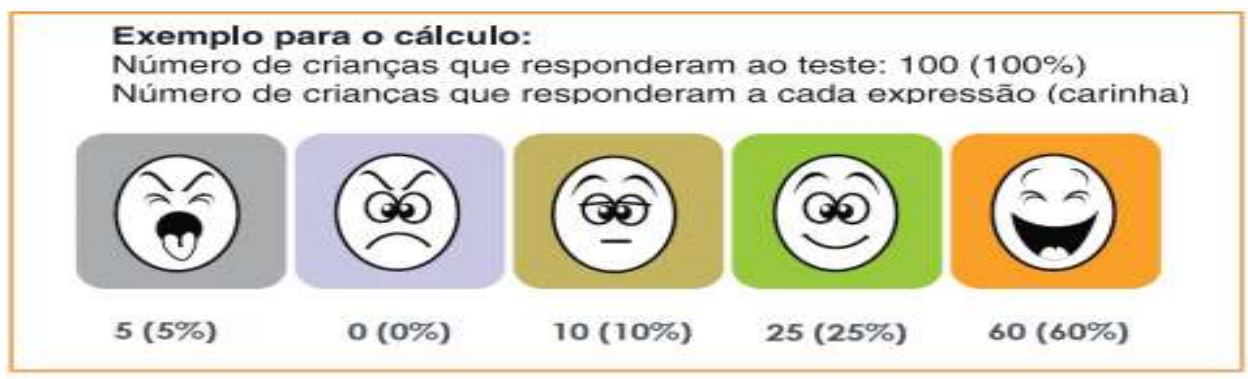

Fonte: FNDE (2017)

Para analisar a resposta do teste observou-se qual amostra em questão apresentou uma percentagem maior ou igual a 85\% nas expressões "gostei” e "adorei", conforme a Figura 2, caso afirmativo, a preparação/alimento testado foi aceito (FNDE 2017).

Figura 2 - Cartelas Lúdicas “gostei” e "adorei’.

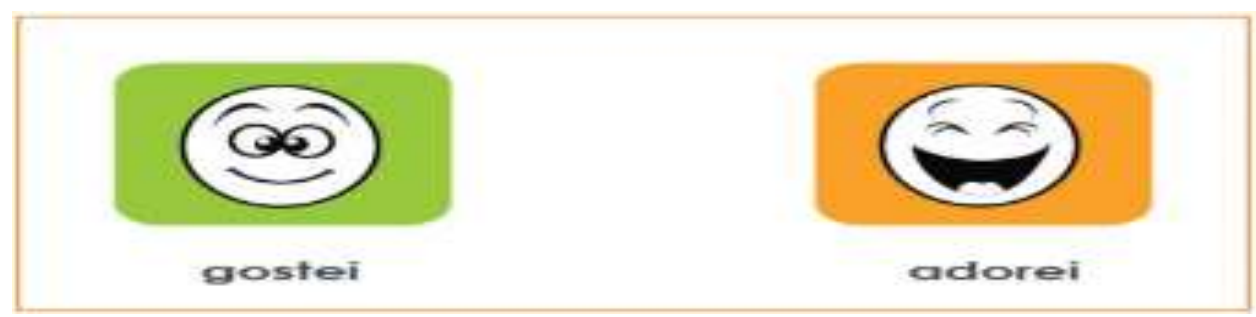

Fonte: FNDE (2017). 
Objetivando o melhor desempenho e aperfeiçoamento do PNAE é indispensável apurar o índice de adesão dos escolares ao Programa, que condiz à grandeza percentual de estudantes que mencionaram consumir a alimentação produzida pelo Centro de Processamento da Merenda Escolar (CEPAE). Na intenção de contribuir a realização da investigação do índice de adesão, aconselha-se que o índice supramencionado seja efetuado no dia da execução do teste de aceitabilidade, sendo necessário a utilização da fórmula presente na Figura 3 (FNDE 2017).

Figura 3 - Cálculo para índice de adesão.

$$
\text { Índice de Adesão }=\frac{\begin{array}{l}
\text { № de estudantes que } \\
\text { consumiram a refeição }
\end{array} \times 100}{\text { № de estudantes presentes na escola }}
$$

Fonte: FNDE (2017).

A vista da conveniência de instituir um critério de referência, o PNAE aplica como ponto de corte os achados atingidos em uma pesquisa realizada pela faculdade de engenharia de alimentos da Universidade de Campinas. Neste estudo a aprovação dos escolares foi avaliada expondo percentuais elencados em quatro conjuntos: alto (acima de 70\%), médio (50 a 70\%), baixo (30 a 50\%) e muito baixo (menor que 30\%) (FNDE 2017).

\subsection{Aspectos éticos}

Pela razão de abordar uma pesquisa com seres humanos, a coleta de dados exigiu o termo de comparecimento assinado pela diretoria das unidades educacionais municipais e pela nutricionista lotada na entidade executora. Segundo o Resolução $\mathrm{n}^{\circ}$ 06, de 08 de maio de 2020 do Fundo Nacional de Desenvolvimento da Educação - FNDE que dispõe sobre o atendimento da alimentação escolar aos alunos da educação básica no âmbito do Programa Nacional de Alimentação Escolar - PNAE, o Art. 20 explica que a EEx deve aplicar teste de aceitabilidade aos estudantes sempre que introduzir no cardápio alimento novo ou quaisquer outras alterações inovadoras, no que diz respeito ao preparo, ou para avaliar a aceitação dos cardápios praticados frequentemente, ainda assim, o seguinte parágrafo único diz que a mesma é responsável pela aplicação do teste de aceitabilidade, o qual deve ser planejado e coordenado pelo RT do PNAE, assim como também para a avaliação antropométrica, conforme metodologia pré-definida (FNDE, 2020).

Não houve nenhum critério de exclusão para a realização da avaliação antropométrica, já para o teste de aceitabilidade foram exclusos alunos que não realizaram o consumo da merenda escolar no dia da pesquisa. Apresentaram-se mínimos os riscos e desconfortos para os participantes que não se enquadraram em nenhum dos critérios de corte e se dispuseram voluntariamente a participar do levantamento de dados. A finalidade da avaliação antropométrica trará um amplo entendimento não só das condições de saúde das crianças, mas também do seu impacto direto sobre desenvolvimento, nível de aprendizagem, grau de retenção e também na evasão escolar.

O intuito do teste de aceitabilidade é servir de ferramenta que pretende garantir a oferta de uma alimentação saudável e adequada, que certifique o atendimento das necessidades nutricionais dos alunos durante o período letivo e atue como um componente pedagógico, caracterizando uma importante ação de educação alimentar e nutricional. Assim, o planejamento dos cardápios, bem como o acompanhamento de sua execução, devem estar aliados para o alcance do objetivo do PNAE. Os dados obtidos nas pesquisas são altamente sigilosos e são utilizados apenas para fins de pesquisa para identificação de desconformidades nos cardápios e agravos na saúde dos escolares, não sendo necessária a divulgação de quaisquer dados pessoais dos alunos (FNDE, 2020). 


\section{Resultados e Discussão}

\subsection{Avaliação antropométrica}

\subsubsection{Ensino fundamental 1}

Pode-se observar na Figura 4, o percentual de alunos em cada classificação (baixo, adequado e elevado) de acordo com o índice peso para idade dos escolares avaliados. Ele mostra que a maioria dos alunos se encontram na classificação "adequada", tanto no turno matutino como no vespertino, com valores de 75,7\% e 86,8\%, respectivamente. Vale ressaltar, também, a presença acentuada de alunos com a classificação elevada para esse mesmo índice, onde obteve-se 22,9\% para os alunos do turno matutino e $13,2 \%$ do turno vespertino. E apenas 1 criança $(1,4 \%)$ foi classificada com o peso baixo para a idade. Nos dados do estudo de Cordeiro, Pereira e Ibiapina (2020), realizado em escolares na rede privada em Teresina - PI, o índice peso/idade obteve maior resultado na classificação "adequado", com alguns alunos em "elevado", e apenas 1 aluno em "baixo", o que corrobora com os achados do presente estudo.

Figura 4 - Diagnóstico nutricional percentual do índice peso para idade dos escolares.

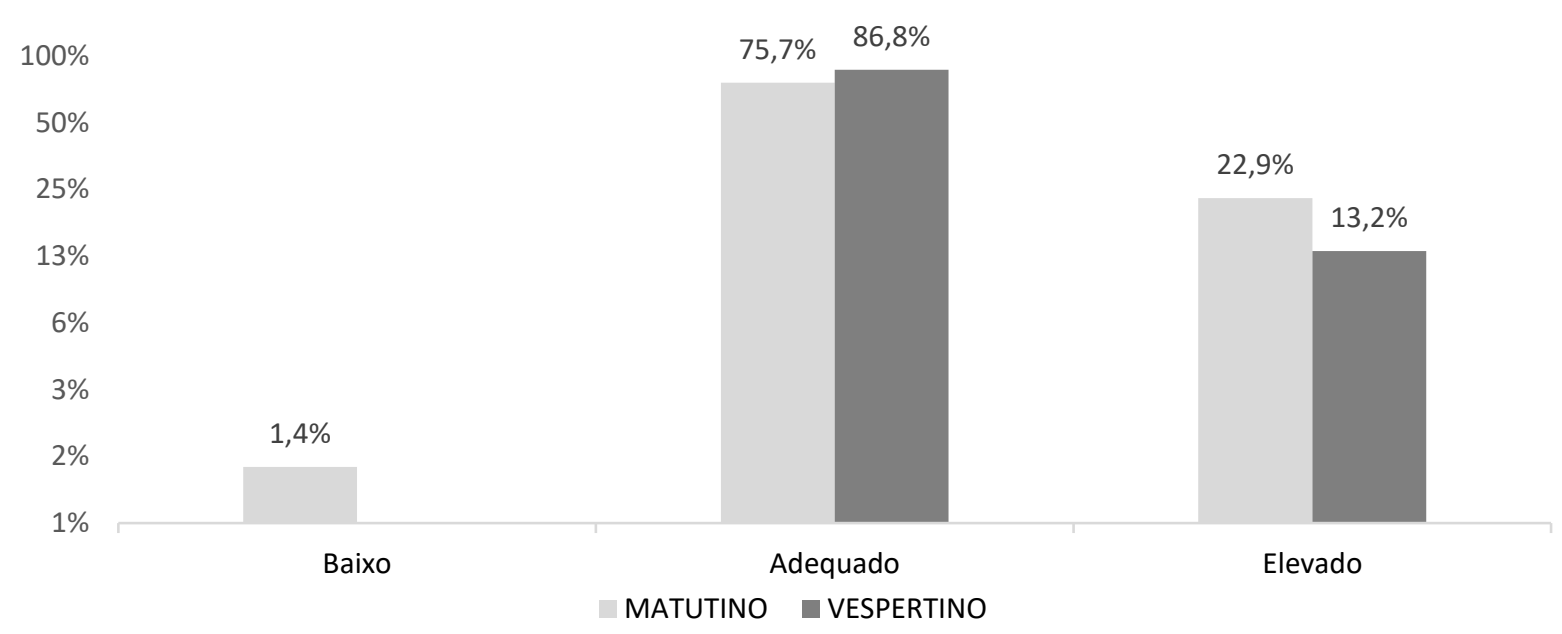

Dados expressos em porcentagem tabulados em Excel após interpretação antropométrica no software "Antro Plus". Matutino (n=70); Vespertino (n=38). Fontes: Autores (2021).

Na Figura 5, o índice avaliado é o de estatura para idade. E percebe-se que a maioria dos alunos se encontra na classificação "adequada", sendo $100 \%$ para o turno matutino e $96,6 \%$ para o vespertino. Ao verificar as outras classificações, encontramos alunos do turno vespertino nas classificações "elevado" e "baixo", com percentuais de 13,2\% e 3,4\%, respectivamente.

O estudo de Pedraza, Silva, Melo, Araújo e Sousa (2017), realizado com escolares da rede pública de Campina Grande - PB, segue o mesmo raciocínio encontrado nesse estudo. A maioria dos alunos $(97,6 \%)$ apresentou classificação adequada, ou seja, a altura adequada para a idade, porém, o restante $(2,4 \%)$ estava com a altura baixa para a idade. Cordeiro et al. (2020) encontraram em sua pesquisa, uma maior quantidade de alunos com a altura adequada para a idade, 3 alunos com a altura elevada para a idade e 1 aluno com baixa altura para idade. Por sua vez, Steil e Poll (2017) avaliaram escolares de duas escolas municipais no interior do município de Candelária - RS, e observaram que todos possuíam altura adequada para a idade. 
Figura 5 - Diagnóstico nutricional, percentual do índice estatura para idade dos escolares.

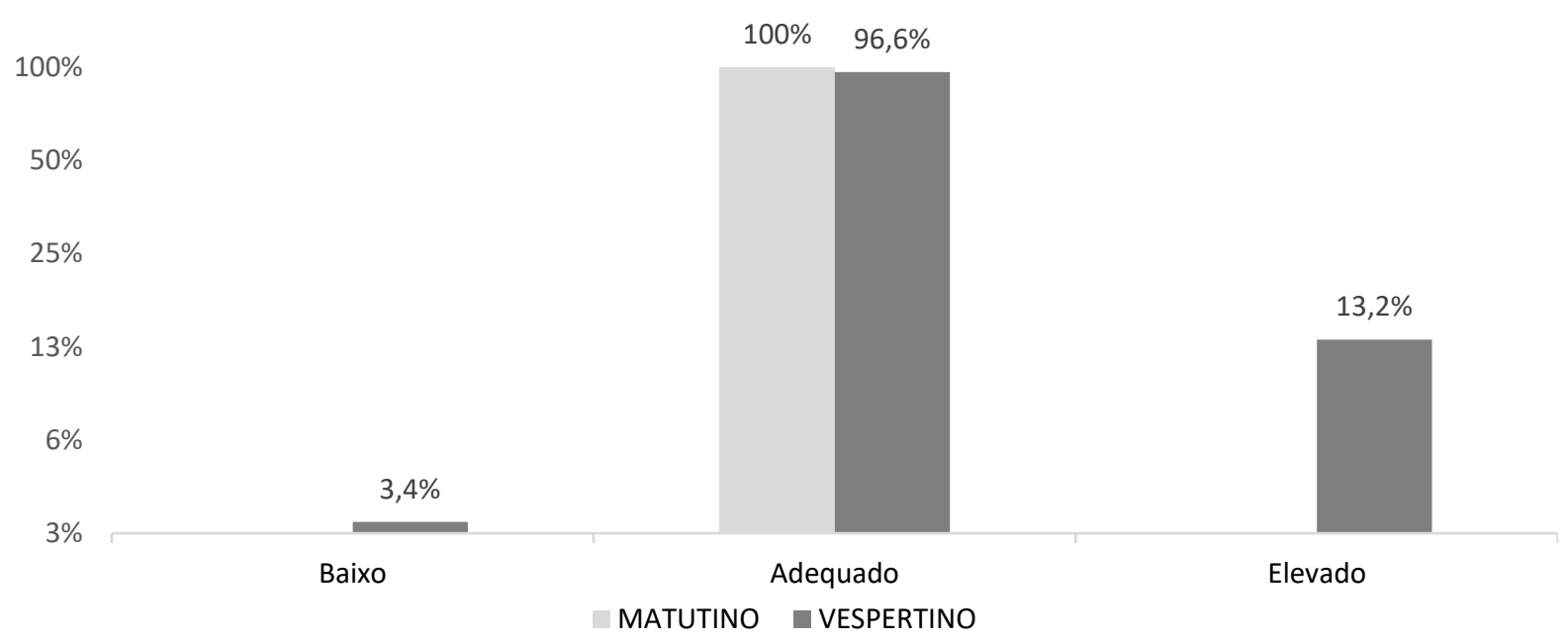

Dados expressos em porcentagem tabulados em Excel após interpretação antropométrica no software "Antro Plus". Matutino (n=97); Vespertino ( $\mathrm{n}=58)$. Fontes: Autores (2021).

No que se refere ao índice IMC para idade, pode-se avaliar a Figura 6. Nela obtém-se que 59\% dos escolares do turno matutino e $71 \%$ do vespertino, estavam na classificação “adequada”. Ademais, vale ressaltar a quantidade de alunos que estavam em excesso de peso (sobrepeso e obesidade), $37 \%$ para os alunos do turno matutino e $26 \%$ para o vespertino.

Figura 6 - Diagnóstico nutricional, percentual do índice IMC para idade dos escolares.

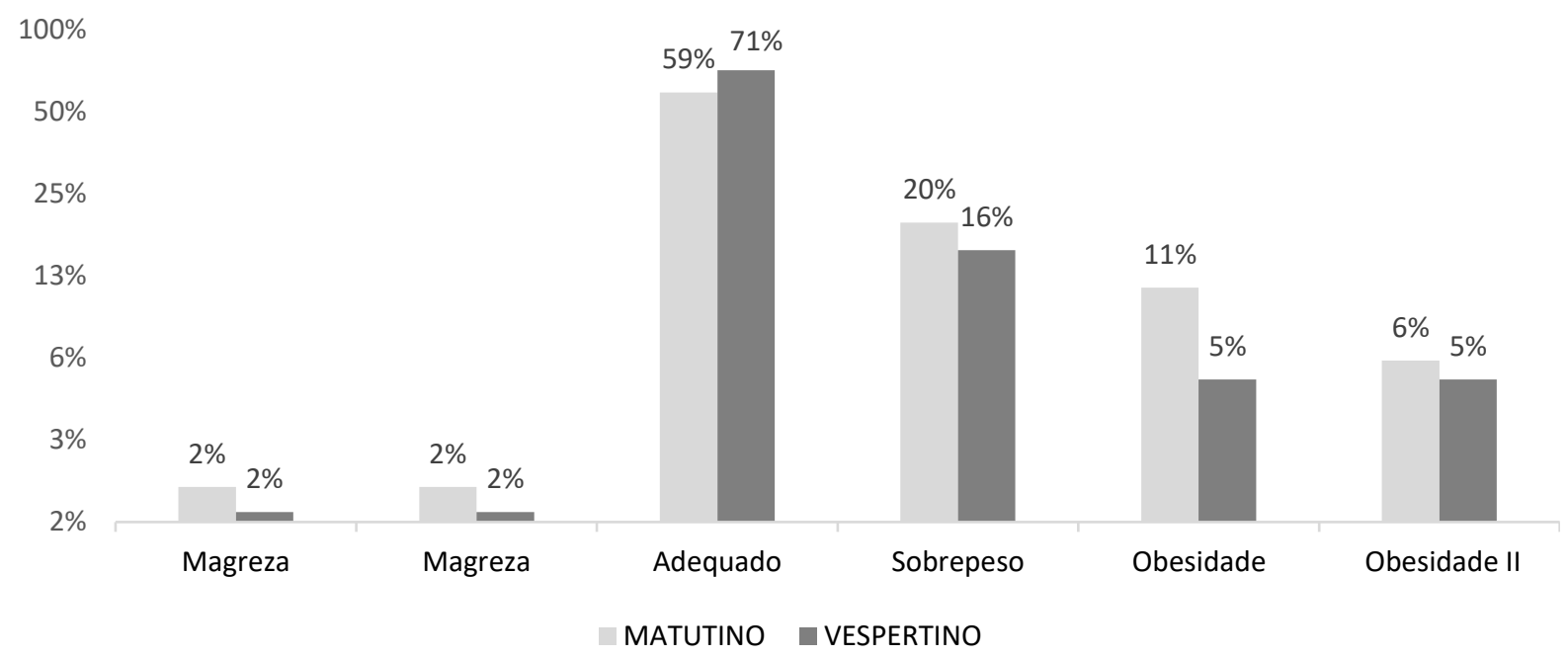

Dados expressos em porcentagem tabulados em Excel após interpretação antropométrica no software "Antro Plus". Matutino (n=97); Vespertino (n=58). Fontes: Autores (2021).

Quando comparamos com estudos que avaliaram o índice em questão, é possível observar uma maior prevalência de eutrofia, porém com valores consideráveis de escolares com peso elevado, como pode ser visto a seguir:

Em uma análise observacional transversal, investigação de campo descritiva, exploratória, quantitativa, realizada com 105 crianças de 7 a 10 anos, de ambos os sexos, onde foram coletados dados antropométricos e observou-se que 18,1\% (19) estavam com sobrepeso, 7,6\% (8) com obesidade e 5,7\% (6) com obesidade grave, 2,9\% (03) com magreza e 2,9\% (03) com magreza acentuada, concluindo que os escolares apresentaram desenvolvimento de sobrepeso, obesidade e excesso de gordura abdominal possuindo relação com riscos de doenças cardiovasculares (Landim et al., 2020). 
Viana, Fumagalli e Ilha (2020), avaliaram escolares do $6^{\circ}$ ao $9^{\circ}$ ano, em uma escola pública estadual de tempo parcial de um município da fronteira oeste do Rio Grande do Sul, e viram que 56,7\% dos alunos estavam com o estado nutricional eutrófico e 42,58\% em sobrespeso/obesidade. As meninas apresentaram resultados superiores aos meninos, pois ocorre um aumento progressivo nos percentuais de gordura, já nos meninos, o percentual de gordura vai diminuindo ao longo das idades.

Steil e Poll (2017) em seu estudo, observaram que 58,2\% dos alunos avaliados estavam em eutrofia e 38,2\% em excesso de peso. Pedraza et al. (2017) encontram que 76,3\% dos escolares apresentaram IMC adequado para idade, 2,2\% com IMC baixo para idade e 21,5\% com IMC de sobrepeso e obesidade. A pesquisa de Cordeiro et al. (2020), segue a mesma linha, a maioria dos participantes se encontram na classificação de eutrofia, uma parcela $(24,4 \%)$ apresentava excesso de peso (sobrepeso e obesidade). Costa et al. (2018), encontraram nos escolares uma prevalência de eutrofia e uma parcela considerável $(24,5 \%)$ apresentando excesso de peso.

\subsubsection{Creche}

A Figura 7 traz os resultados dos indicadores de peso para idade dos escolares da creche, nele pode ser observado que a maioria dos alunos apresentou níveis adequados para essa classificação do estado nutricional, com um total de $67,2 \%$ no período matutino e 65,6\% no período vespertino, não apresentando diferença observável entre turnos. Porém, ao considerar as demais classificações, é válido observar a obtenção de níveis de até $25,0 \%$ na quantidade de escolares acima do peso adequado para sua respectiva idade, sobretudo no turno da manhã.

Figura 7 - Diagnóstico nutricional, percentual do índice peso para idade dos escolares.

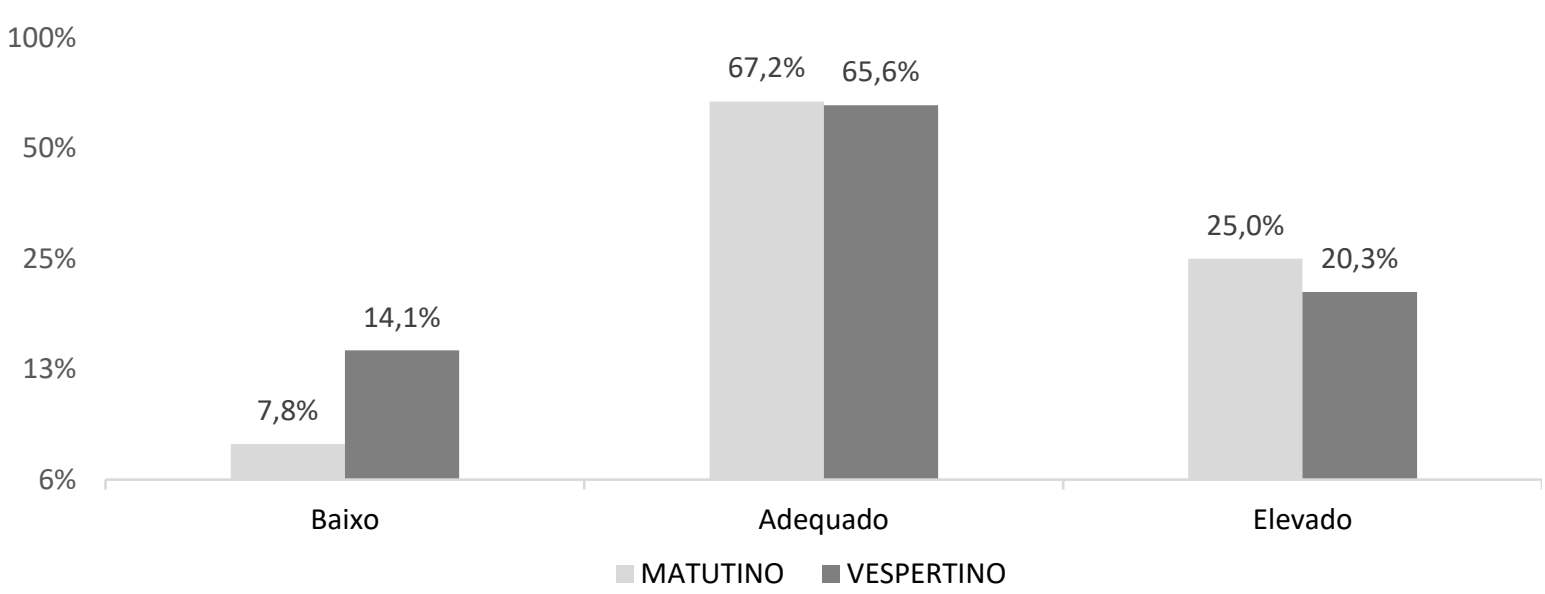

Dados expressos em porcentagem tabulados em Excel após interpretação antropométrica no software "Antro Plus". Matutino (n=64); Vespertino ( $\mathrm{n}=64)$. Fontes: Autores (2020).

Os níveis mais elevados de restrição alimentar impostos por familiares estão associados a níveis mais elevados de altura e peso das crianças percepcionados pelos pais. No que diz respeito à relação entre a restrição e altura, esta poderá ser explicada por outras variáveis, nomeadamente o estado ponderal dos pais, possível histórico de problemas alimentares e com o peso, percepção de desvios de peso ou de risco, bem como preocupação de que a criança venha a apresentar problemas de peso (Santos et al., 2020).

Quanto a classificação do indicador estatura para idade, foi visto que, mais uma vez a grande maioria dos escolares estava na faixa de adequação, 78,1\% no turno da manhã e 73,4\% à tarde (ver Figura 8) e, novamente, sem muita diferença observável entre turnos. Vale ressaltar que, mesmo com altas porcentagens de eutrofia, os níveis de baixa estatura para idade, atingiram percentuais de até $17,2 \%$, novamente, sobressaindo-se o turno da manhã. 
Dados semelhantes, porém mais elevados, foram encontrados em escolares matriculados em creches, com relação aos valores adequados de P/I e E/I, foram encontrados no estudo de Ferreira (2017), apresentando um total de 96,7\% e 98\%, respectivamente, utilizando como material norteador, as orientações do SISVAN para coleta e análise de dados antropométricos. Medeiros (2016), por meio do WHO Anthro Plus, também encontrou resultados positivos quanto a esses dois indicadores - P/I e E/I - trazendo respectivamente, os valores para adequados os percentuais de 98,4\% e 100,0\%. Porém, em divergência aos dados encontrados no presente estudo, na análise dos dados da autora, não haviam crianças com E/I abaixo do recomendado, e cerca de $1,6 \%$ indicava possível sobrepeso.

Figura 8 - Diagnóstico nutricional, percentual do índice estatura para idade dos escolares.

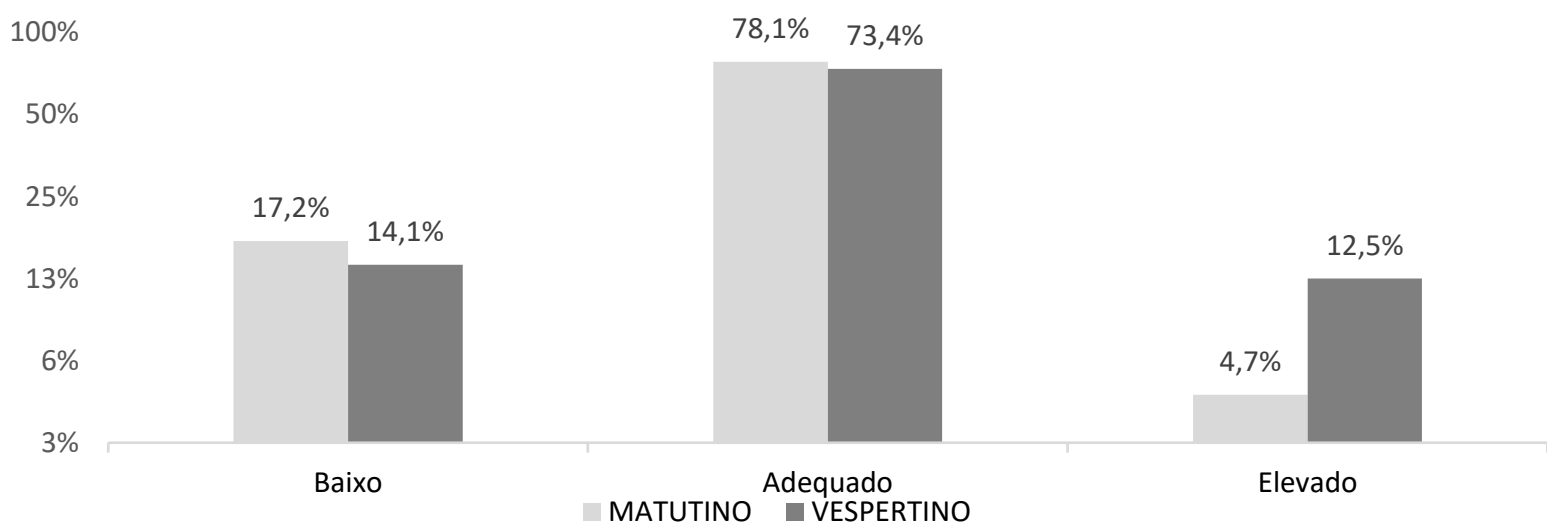

Dados expressos em porcentagem tabulados em Excel após interpretação antropométrica no software "Antro Plus". Matutino (n=64); Vespertino $(\mathrm{n}=64)$. Fontes: Autores.

Diferentemente do trabalho em questão, os dois estudos apresentaram porcentagens inferiores de classificações com relação aos indicadores "abaixo" e "acima" do recomendado para as faixas etárias, e assemelham-se quanto as maiores porcentagens de adequação, tornando a possível prevalência de escolares com estado nutricional adequado, quanto a esses parâmetros. Contudo, não é negada a importância e necessidade de intervenção direcionada as crianças, principalmente as que se encontram em risco nutricional. Vale lembrar que analisar apenas um ou outro indicador não é suficiente para determinar a condição nutricional, é preciso um apanhado de informações, por isso são citados como possibilidades.

No que se refere aos valores de peso para estatura é importante salientar que os mesmos não contemplam o total de $100 \%$ em nenhum dos turnos, pelo fato de não haver classificação para esse indicador para crianças com faixa etária acima dos 5 anos de idade, e como dito, a creche atende crianças até os 6 anos de idade, portanto, os valores descritos contemplam as crianças que correspondiam a faixa etária que se encaixa ao indicador. Em relação ao parâmetro supracitado, destacam-se as classificações "adequado" e "elevado". Obteve-se nos escolares do período matutino os valores de 35,9\% e 20,3\%, respectivamente, e 31,3\% para adequado e 14,1\% para elevado, no período vespertino, como podemos ver na Figura 9. 
Figura 9 - Diagnóstico nutricional, percentual do índice peso para estatura dos escolares.

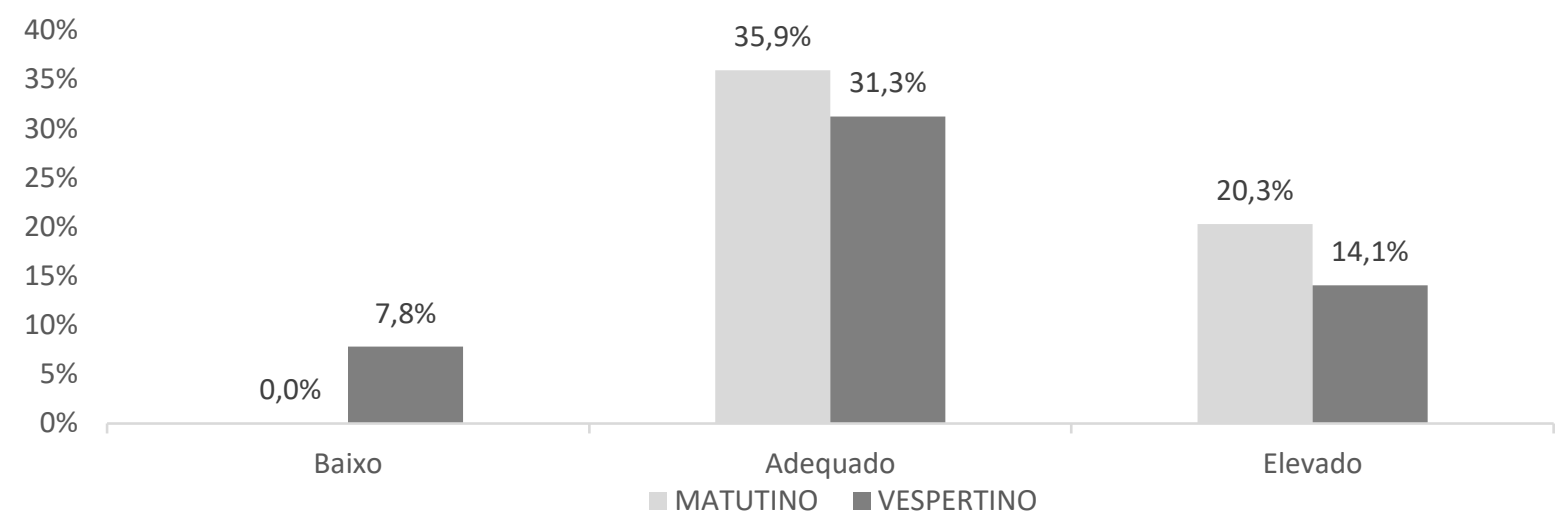

Dados expressos em porcentagem tabulados em Excel após interpretação antropométrica no software "Antro Plus". Matutino (n=64); Vespertino (n=64). Fontes: Autores.

Os estudos de Ferreira (2017) e Medeiros (2016), ainda trouxeram os dados de P/E dos escolares, também com maior destaque para o estado de adequação, sendo respectivamente, $85 \%$ e 44,3\%, este último com valor mais aproximado ao da pesquisa em questão - 35,9\% e 31,3\%. Mesmo com porcentagens inferiores a classificação de "eutrofia”, o P/E “elevado" foi mais acentuado que o "baixo" nos estudos antes citados, chegando a até 11,5\% no estudo de Ferreira (2017), sendo classificado como risco para sobrepeso dentro desse indicador.

De forma a contextualizar os três indicadores aqui discurtidos, o panorama literário sobre avaliação antropométrica em creches feito por Pedraza e Menezes (2016), que avaliou dados de estudos desde 1990 até períodos posteriores a 2010, trouxe que os dados de P/I, E/I e P/E foram usados para diagnóstico do estado nutricional em alguns dos estudos analisados. Em determinados locais como São Paulo, Minas Gerais e Pará, no que diz respeito ao baixo P/I, os resultados variaram, podendo chegar até 53\%; quanto a baixa E/I, podem chegar a aproximadamente 55\%; já com relação ao P/E, os quadros de desnutrição aguda vão de 0 a 5\%. Dados posteriores a 2010 nos estudos analisados, demonstraram variações entre o sobrepeso segundo o $\mathrm{P} / \mathrm{E}$, a desnutrição aguda e o déficit de estatura, com valores até 9,8\%, 1,8\% e 8,6\%, respectivamente.

\subsection{Teste de aceitabilidade}

\subsubsection{Ensino fundamental 1}

Dentre os alunos matriculados na escola, fazendo parte do ensino fundamental I, 88 alunos consumiram a preparação (risoto de carne com soja) e participaram da pesquisa. O estudo avaliou a aceitação dos cardápios através da frequência de votação da escala hedônica facial, sendo que 11 alunos (12,5\%) levantaram a mão quando foi mostrada a placa com a carinha “detestei”, 10 alunos (11,3\%) quando mostrou a carinha "não gostei”, 8 alunos (9\%) se identificaram com a carinha "indiferente", 23 alunos $(26,1 \%)$ gostaram da preparação e 36 alunos (40\%) adoraram a preparação.

Através dos resultados obtidos pôde-se observar que a amostra apresentou uma percentagem menor que $85 \%$ nas expressões "gostei” e "adorei”, logo a preparação/alimento testado não foi bem aceito. Com relação ao índice de adesão, observou-se que a amostra apresentou uma percentagem entre 50 e $70 \%$, logo a preparação/alimento testado obteve nível médio de adesão. Pode-se observar os cálculos a partir do Quadro 1. 
Quadro 1 - Cálculos de percentual de aceitação e adesão.

\begin{tabular}{|l|l|}
\hline Índice de aceitação & $\underline{23+36}=67,05 \%$ \\
\hline Índice de adesão & $\underline{88 \times 100}=53,3 \%$ \\
\hline
\end{tabular}

Fonte: Autores (2020).

Os pesquisadores Avila e Pinheiro (2019), realizaram um teste de aceitabilidade com 81 escolares numa Escola Municipal de Ensino Integral da cidade de Pinheirinho do Vale - RS, com duas preparações, uma vitamina de banana e um bolo de cenoura, ambas com a presença de cacau, pensando na inclusão das preparações na alimentação escolar. Foi observado que a vitamina de banana com cacau e o bolo de cenoura com cacau, apresentaram grandes porcentagens em "gostei" e "adorei", obtendo um índice de aceitabilidade de $91,3 \%$ e $87,6 \%$, respectivamente, sendo considerada adequada para inclusão na alimentação escolar.

Os testes de aceitabilidade são de grande importância entre os escolares, tendo em vista que é uma ferramenta para avaliar a qualidade sensorial da alimentação ofertada, ou seja, como está a interação dos escolares com o alimento oferecido.

\subsubsection{Creche}

Com a adaptação do teste de aceitabilidade, ao questionar o motivo pelo qual algumas crianças não se serviam ou por relatar o não agrado pela refeição em questão, foi visto que a maioria das queixas, direcionavam-se ao gosto da soja, da mistura em si (arroz com soja) e da presença dos condimentos coentro e cebola, principalmente por estarem visíveis na preparação. Um exemplo disso, foi o estudo de Woyniak e Ghisleni (2012), que tratou de uma avaliação de aceitabilidade de preparações padrão de alimentos, como bolos salgados, hambúrgueres, pizzas e pastéis, com substituição total ou parcial da carne por proteína texturizada de soja, onde a preparação que teve menor aceitação (não alcançou o índice mínimo preconizado), foi o hambúrguer, e atribuíram a possibilidade de ocorrência desse fato, ao alimento ter sido preparado com a substituição supracitada, porém traz também que a pizza e o pastel contaram com essa substituição total, contudo com o acréscimo de alguns condimentos ao preparo. Notando-se assim que, o sabor da soja continua sendo uma das questões mais associadas à sua aceitação ou não aceitação.

Ao observar a baixa aceitação de alguns alimentos e/ou refeições servidos em ambientes escolares, mesmo não sendo unanimidade, Silva e Vieira (2019) reforçam a importância de avaliar os motivos envolvidos na não aceitação ou baixo índice de satisfação dos escolares com relação as refeições oferecidas. Da mesma forma, Silva e Garcia (2018) relatam a possibilidade de realização de mudanças ser possível a partir da verificação do que não está do agrado dos comensais, por meio das respostas negativas aos testes de aceitabilidade

\section{Considerações Finais}

A presente revisão pretendeu avaliar o estado nutricional de escolares e identificar as porcentagens de aceitação das refeições oferecidas em ambientes escolares, com isso conclui-se que, o escolares se apresentam com características da transição nutricional, que é marcada pelo aumento do indicador de sobrepeso/obesidade, tornando-se necessário atentar para o fato de que, mesmo que as porcentagens de eutrofia tenham se sobressaído em comparação as outras classificações, não deve ser descartada a presença de crianças abaixo ou acima do peso e/ou altura recomendados, voltando o olhar para ações de EAN.

O índice de aceitabilidade e de aceitação dos escolares com relação a preparação do risoto de carne com soja, mostrou respectivamente, que a preparação não foi bem aceita e que a preparação testada obteve nível médio de adesão. Da mesma forma que na creche, a preparação do arroz com proteína texturizada de soja, de acordo com as queixas dos escolares, não foi bem 
aceita por características bem marcantes do prato servido, sendo uma delas o próprio sabor da soja, ingrediente principal da refeição.

Faz-se necessário, dessa forma, que o ambiente escolar funcione como veículo fornecedor de informações sobre hábitos alimentares saudáveis, através de atividades educativas, sendo a educação nutricional uma estratégia para esse fim e, também, para promover uma maior aceitação da alimentação escolar, além de avaliar os motivos ou o que não está agradando os comensais, a fim de que possam haver adaptações ou melhorias nos aspectos sensoriais das refeições servidas.

Mais pesquisas na área de alimentação escolar, além de antropometria e análise dos índices de adesão e aceitação devem ser realizados, pois são importantes para identificar o perfil nutricional e avaliar a qualidade sensorial das preparações oferecidas. Deve-se considerar que há uma escassez de estudos com relação a aceitabilidade da merenda escolar, assim, o estudo em questão pode contribuir para pesquisas futuras.

\section{Referências}

Avila, C., \& Pinheiro, T. (2020). Aceitabilidade de preparações com cacau na alimentação escolar. Nutrição Brasil, 18(3), 127. 10.33233/nb.v18i3.2159

Brasil. Ministério da Saúde. (2015). NutriSUS - Estratégia de fortificação da alimentação infantil com micronutrientes (vitaminas e minerais) em pó: manual operacional. http://bvsms.saude.gov.br/bvs/publicacoes/nutrisus_estrategia_fortificacao_alimentacao_infantil.pdf

Brasil. Ministério da Saúde. (2011). Orientações para a coleta e análise de dados antropométricos em serviços de saúde: Norma Técnica do Sistema de Vigilância Alimentar e Nutricional-SISVAN. http://189.28.128.100/dab/docs/portaldab/publicacoes/orientacoes_coleta_analise_dados_antropometricos.pdf

Cordeiro, M. C., Pereira, B. A. D., \& Ibiapina, D. F. N. (2020). Hábito alimentar, consumo de ultraprocessados e sua correlação com o estado nutricional de escolares da rede privada. Research, Society and Development, 9(3), e21932300-e21932300. 10.33448/rsd-v9i3.2300

Costa, M. C., Sousa, A. F., Nascimento Lima, J. T., Sousa, S. D. F., Ferreira, F. V., \& Azevedo Marques, A. R. (2018). Estado nutricional, práticas alimentares e conhecimentos em nutrição de escolares. Revista de Atenção à Saúde, 16(56), 12-17. 10.1590/S0103-05822010000300008

Facchini, A., \& Campagnolo, P. D. B. (2020). Consumo da alimentação escolar e qualidade da dieta de escolares. Revista da Associação Brasileira de Nutrição - RASBRAN, 11(1), 115-127. https://doi.org/10.47320/rasbran.2020.1377

Ferreira, D. T. D. O. (2017). Condição de peso em crianças de creches participantes do Programa Escola com Saúde. Trabalho de conclusão de curso, Universidade Federal de Mato Grosso, Cuiabá, MT, Brasil. https://bdm.ufmt.br/handle/1/90

Ferreira, L. D., Dellandrea, S., Kormann, K., Vargas, D. M., \& Azevedo, L. C. (2020). Um estudo sobre a eficiência da educação nutricional em adolescentes escolares. RBONE - Revista Brasileira de Obesidade, Nutrição e Emagrecimento, 14(85), 199-206. http://www.rbone.com.br/index.php/rbone/article/view/1229

Fundo Nacional de Desenvolvimento da Educação (FNDE). Manual para aplicação dos testes de aceitabilidade no Programa Nacional de Alimentação Escolar (PNAE). http://Teste\%20de\%20\%20 Aceitabilidade\%20_\%202a\%20edicao\%202017.pdf

Fundo Nacional de Desenvolvimento da Educação - FNDE. (2020). Programa Nacional de Alimentação Escolar. Resolução No 06, De 08 De Maio De 2020. https://www.fnde.gov.br/index.php/acesso-a-informacao/institucional/legislacao/item/13511-resolu\%C3\%A7\%C3\%A3o-n\%C2\%BA-6,-de-08-de-maio-de2020

IBGE. Instituto Brasileiro de Geografia e Estatística. (2020). Área Territorial Estimada. Resolução da Presidência do IBGE de n 5 (R.PR-5/02). Recuperado em: https://www.ibge.gov.br/cidades-e-estados/pb/picui.html?

IBGE. Instituto Brasileiro de Geografia e Estatística. (2019). Estimativa populacional. https://cidades.ibge.gov.br/brasil/pb/picui/panorama.

LandimL. A. dos S. R., CordeiroM. C., BarbosaA. M., SeveroJ. S., IbiapinaD. F. N., \& PereiraB. A. D. (2020). Avaliação nutricional, consumo alimentar e frequência de ultraprocessados em escolares da rede pública. Revista Eletrônica Acervo Saúde, 12(5), e2427. https://doi.org/10.25248/reas.e2427.2020

Medeiros, M. S. (2016). Avaliação do estado nutricional dos pré-escolares de uma creche no município de Santa Cruz-RN (Bachelor's thesis, Universidade Federal do Rio Grande do Norte). https://monografias.ufrn.br/jspui/handle/123456789/2374

Oliveira, T. C., Albuquerque, I. Z., Stringhini, M. L. F., Mortoza, A. S., \& Morais, B. A. (2017). Estado nutricional de crianças e adolescentes hospitalizados: comparação entre duas ferramentas de avaliação nutricional com parâmetros antropométricos. Revista Paulista de Pediatria, 35(3), 273-280. https://doi.org/10.1590/1984-0462/,2017,35,3,00006

OMS. Organização Mundial de Saúde. (2006,2007). Growth reference data for 0-5 and 5-19 years. http://www.who.int/childgrowth/en/ e http://www.who.int/growthref/en/

Pedraza, D. F., \& de Menezes, T. N. (2016). Caracterização dos estudos de avaliação antropométrica de crianças brasileiras as sistidas em creches. Revista Paulista de Pediatria, 34(2), 216-224.

Pedraza, D. F., Silva, F. A., Melo, N. L. S. D., Araujo, E. M. N., \& Sousa, C. P. D. C. (2017). Estado nutricional e hábitos alimentares de escolares de Campina Grande, Paraíba, Brasil. Ciência \& saúde coletiva, 22, 469-477. 10.1590/1413-81232017222.26252015 
Pereira, A. S. et al. (2018). Metodologia da pesquisa científica. UFSM. https://repositorio.ufsm.br/bitstream/handle/1/15824/Lic_Computacao_MetodologiaPesquisa-Cientifica.pdf?sequence $=1$

Prodanov, C. C., \& de Freitas, E. C. (2013). Metodologia do trabalho científico: métodos e técnicas da pesquisa e do trabalho acadêmico-2a Edição. Editora Feevale.

Rocha, N. P., Filgueiras, M. D. S., Albuquerque, F. M. de, Milagres, L. C., Castro, A. P. P., Silva, M. A., Costa, G. D. da, Priore, S. E., \& Novaes, J. F. (2018). Análise do programa nacional de alimentação escolar no município de Viçosa, estado de Minas Gerais. Revista De Saúde Pública, 52, 16. https://doi.org/10.11606/S1518-8787.2018052007090

Rocha, T., \& Etges, B. (2019). Consumo De Alimentos Industrializados E Estado Nutricional De Escolares. Biológicas \& Saúde, 9(29). $10.25242 / 886892920191402$

Santos, A. F., Fernandes, C., Cardia, S., Fernandes, M., Bost, K. K., \& Veríssimo, M. (2020). Concordância entre dados antropométricos reportados vs. medidos e relação com as práticas parentais alimentares em idade pré-escolar. Análise Psicológica, 38(1). https://core.ac.uk/download/pdf/324203637.pdf

Silva, J. D. D., \& Garcia, P. P. C. (2018). Avaliação e aceitabilidade do cardápio da alimentação escolar de uma creche pública no DF. Revista Científica. v. 1 n. 1 (2019). Recuperado em: http://189.112.117.16/index.php/revista-cientifica/article/view/175

Silva, T. F., \& Vieira, V. B. R. (2019). Adesão e Aceitabilidade de Preparações do Cardápio de uma Escola Municipal do Interior do Estado de São Paulo/Sp. Revista Científica, 1(1). http://189.112.117.16/index.php/revista-cientifica/article/view/175

Souza, J., dos Santos, R. B., Cavalheiro, M. P., Soares, R. C., Sommer, R. M., \& Dos Santos, L. P. (2017). Comparação da Classificação do Estado Nutricional Realizadas Manualmente e Utilizando o Anthro e Anthro Plus. Anais do Salão Internacional de Ensino, Pesquisa e Extensão, 9(2). Recuperado de https://periodicos.unipampa.edu.br/index.php/SIEPE/article/view/98816.

Souza, V. R., Ferreira, I., Leão, D. P., Barbosa, K. J. F., Guimarães, F. M. T., \& Silva, A. R. A. (2020). Avaliação do Estado Nutricional de Escolares de uma Escola da Zona Leste da Cidade de Manaus. BIUS-Boletim Informativo Unimotrisaúde em Sociogerontologia, 16(10), 1-13. https://periodicos.ufam.edu.br/index.php/BIUS/article/view/7168

Steil, W., \& Poll, F. (2017). Estado nutricional, práticas e conhecimentos alimentares de escolares. Cinergis, 18(3), 226-232. 10.17058/cinergis.v18i3.9385

Sturion, G. L. (2002). Programa de alimentação escolar: avaliação do desempenho em dez municípios brasileiros. http://repositorio.unicamp.br/jspui/handle/REPOSIP/254938

Universidade Federal de São Paulo-UNIFESP - Centro Colaborador de Alimentação e Nutrição do Escolar da (CECANE). (2010). Manual para Aplicação dos Testes Aceitabilidade no Programa Alimentação Nacional de https://www.unifesp.br/campus/san7/images/cecane/manual_aplicacao_testes_aceitabilidade_programa_nacional_alimentacao_escolar.pdf

Viana, R., Fumagalli, L., \& Ilha, P. (2020). Estado nutricional e fatores relacionados de escolares. Research, Society And Development, 9(1), 1-14. 10.33448 / rsd-v9i1.1649

WHO. World Health Organization. (2008). child growth standards: training course on child growth assessment. https://apps.who.int/iris/bitstream/handle/10665/43601/9789241595070_B_eng.pdf.

Woyniak, R. P., \& Ghisleni, C. P. (2012). Aceitação da proteína texturizada de soja em uma escola municipal rural de Barão de Cotegipe, RS. https://www.uricer.edu.br/cursos/arq_trabalhos_usuario/3298.pdf 